\title{
Productores de la parroquia "Los Andes-Patate" buscan dar valor agregado al durazno
}

\author{
"Los Andes-Patate" county seek for adding value to peach production
}

\section{Mishel Viviana Viteri Tipán ${ }^{(a)}$, Norman Aurelio Soria Idrovo ${ }^{\left({ }^{*} a\right.}$}

(a) Universidad de las Fuerzas Armadas - ESPE, Sangolquí-Ecuador

${ }^{(*)}$ nasoria@espe.edu.ec

\section{Resumen}

El durazno (Prunus persica L.) es una fruta que posee un atractivo color y sabor, características por las cuales se cultiva a nivel mundial, incluido el Ecuador. El cantón Patate posee una representativa producción de durazno por lo cual nace la importancia de realizar vinos. El objetivo del estudio es desarrollar un protocolo efectivo en la elaboración de vino de durazno de bajo contenido alcohólico, en el estudio se utilizaron tres variedades de durazno: Criollo, Pepa de Oro, y Abridor "Rosado de Sevilla"; que fueron inoculadas con cepas de levaduras: Safale S-04, Cider Yeast, MO2, y conservadas con procesos térmicos y de carbonatación. El proceso de fermentación duró aproximadamente 15 días y el producto final fue un vino de mesa con un grado alcohólico que no excedía los $12^{\circ} \mathrm{GL}$, los tratamientos que contenían las variedades Abridor y Pepa de Oro tuvieron una buena clarificación, mientras que con la variedad Criollo el proceso de clarificación fue lento. Se obtuvo un menor contenido de alcohol con la variedad Pepa de Oro; cepas Cider Yeast y MO2; y conservación térmica. Al final del proceso de elaboración se realizó un análisis sensorial de los vinos, obteniéndose que los tratamientos que correspondían a la variedad Abridor, las cepas Safale S-04 y Cider Yeast, y con la conservación carbonatada fueron los que consiguieron una mejor calificación global. La producción artesanal de vino es una alternativa para dar valor agregado al cultivo de durazno.
Palabras clave: Durazno, vino, cepas de levadura, grado alcohólico, variedades de durazno.

\section{Abstract}

The peach (Prunus persicaL.) is a fruit that has an attractive color and flavor. It is grown worldwide including in Ecuador. Patate county is an important producer of peaches and they seek to add value to their production by making wine. The present study developed an effective protocol for producing artisanal peach low alcohol wine. Three varieties of peaches were used: Criollo, Pepa de Oro, Abridor "Rosado de Sevilla"; they were inoculated with yeast strains: S-04 Safale, Cider Yeast, MO2, and then treated with heat and carbonation processes. The fermentation process lasted approximately 15 days. The final product was a wine with an alcohol content under $12^{\circ} \mathrm{GL}$. Treatments containing the Abridor and Pepa de Oro varieties had a good clarity. Lower alcohol content was obtained with the variety Pepa de Oro, Cider Yeast strains, MO2, and thermal storage. A sensory analysis of the produced wine was made, the treatment with the variety Abridorsx, strains Safale S-04, Cider Yeast, and carbonated got the best overall rating.

Key words: Peach, wine, yeast strains, alcoholic grade, varieties of peach. 


\section{Introducción}

El cantón Patate es una zona conocida por su alta productividad y gran variedad de frutales; algunos de sus productos cultivados se comercializan con valor agregado como la famosa "chicha de uva y arepas patateñas" mismas que son aceptadas en el mercado por su palatabilidad y calidad. Los limitados conocimientos en la elaboración de productos con valor agregado, hablando de la conservación de bebidas alcohólicas, no ha permitido prosperar su producción, a su vez, se presenta la falta de valor agregado a las materias primas producidas en la parroquia Los Andes, como las variedades de durazno locales, las cuales son vendidas a bajos precios por los productores a los intermediarios y son ofertados nuevamente en los mercados a precios más elevados, lo que provoca que los consumidores no compren el producto nacional, es por ello que los productores de frutas de la parroquia Los Andes necesitan capacitación y transferencia de tecnología.

Mediante el proyecto de vinculación, capacitación y transferencia de tecnología para reactivar el cultivo duraznero en Los Andes - Patate, (CTTLA); la Universidad de las Fuerzas Armadas ESPE apoya los productores con la elaboración de bebidas de reducido nivel alcohólico con variedades de duraznos cultivadas en la mencionada parroquia.

El durazno (Prunus persica L.) es una fruta que posee un atractivo color y sabor, características por las cuales se cultiva a nivel mundial, incluido el Ecuador. El cantón Patate posee una representativa producción de durazno (PDOT, 2011), por lo cual nace la importancia de realizar distintos productos para su consumo por ejemplo bebidas de reducido nivel alcohólico; el estudio se efectuó para desarrollar un protocolo efectivo en la elaboración y conservación de vino de durazno, utilizando tres variedades de durazno y diferentes cepas de levadura que ayude a dar valor agregado al fruto a través de la producción artesanal. Así se mejora la economía de los fruticultores de los Gobiernos Autónomos Descentralizados (GAD), parroquia Los Andes, Patate. Para este fin se procedió a analizar la eficiencia de tres procesos fermentativos en las bebidas para reducir el nivel alcohólico con diferentes cepas de levadura y posteriormente se evaluaron dos métodos de conservación carbonatado (químico) y térmico (físico) en las bebidas, a través de pruebas de aceptación, al final del tiempo de conservación y luego se difundieron los resultados del protocolo más idóneo o eficiente en la elaboración de bebidas de durazno con bajo contenido de alcohol.

\section{Materiales y métodos}

\subsection{Descripción del sitio de estudio}

La fruta se recolectó en Ecuador, provincia de Tungurahua, cantón Patate, parroquia Los Andes, sector Erapamba a, $1^{\circ} 18^{\prime} 46.83^{\prime \prime}$ (S) y 78 30'23.55" (O) y a una altitud de $2180 \mathrm{msnm}$.

Los ensayos de procesamiento se realizaron en el laboratorio de agroindustrias de la Universidad de las Fuerzas Armadas - ESPE, ubicado en la hacienda El Prado, situada en el Ecuador, Provincia de Pichincha, Cantón Rumiñahui, Parroquia San Fernando a, $0^{\circ} 23^{\prime} 27.98^{\prime \prime}$ (S) y $78^{\circ} 24^{\prime} 49.16^{\prime \prime}$ (O) y a una altitud de $2745 \mathrm{msnm}$.

\subsection{Fase de campo}

Se recolectaron $6 \mathrm{Kg}$. aproximadamente de cada variedad de durazno: Criollo, Pepa de Oro y Abridor "Rosado de Sevilla". Durante la recolección se utilizaron canastas y fundas plásticas. Los frutos recolectados fueron caracterizados por su tamaño.

\subsection{Fase de laboratorio}

Las bebidas se elaboraron con tres variedades recolectadas de durazno (ver Fig 1). Se analizó el proceso de fermentación con dos cepas de levaduras de sidra: Cider Yeast y MO2 cider, y una cepa de cerveza Safale S-04. Para conservar las bebidas de reducido nivel alcohólico se analizaron dos tipos de conservación: térmica y carbonatada.

La cepa de levadura Cider Yeast, se puede utilizar con jugo recién prensado o concentrado, proporciona un agradable sabor y olor a la bebida (Mad Millie, 2011). MO2 cider es un tipo de cepa de levadura que otorga una alta producción de ésteres en la sidra impartiendo una maravillosa profundidad de sabor, revelando al fruto en pleno potencial, (Mangrove Jack`s, 2015). Safale S-04 es una cepa inglesa comercial del tipo Ale, seleccionada por su rápida velocidad de fermentación y la capacidad de formar un sedimento compacto en el fondo de los fermentadores, (Fermentis, 2015) 


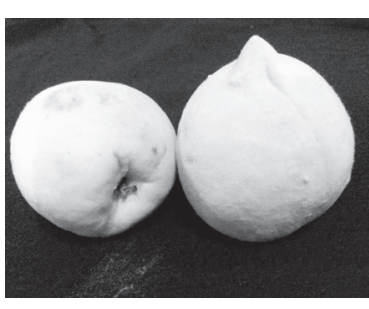

Var. Crioll

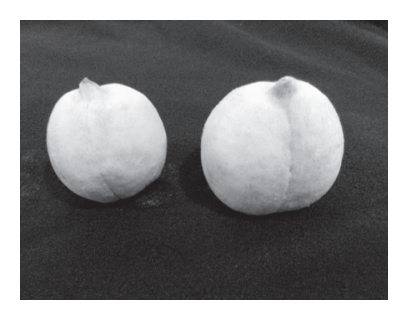

Var. Pepa de Oro

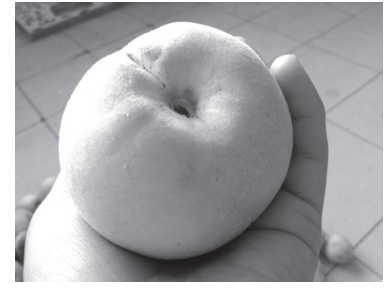

Var. Abridor

"Rosado de Sevilla"
Figura 1. Variedades de durazno, por Viteri y Soria (2015)

Según Pérez y Gervás, (2010) el proceso para la elaboración de las bebidas de bajo grado alcohólico está descrito como sigue: se receptó la materia prima en el laboratorio de procesamiento de vegetales de la carrera de Ingeniería Agropecuaria (IASA I), Hda. El Prado.

Para desarrollar las características organolépticas ideales, se colocaron los frutos en un cuarto cerrado con mínima luz y sin el contacto entre los mismos para no estimular una sobre maduración, durante 4 días. Los frutos muy dañados o golpeados fueron eliminados y los frutos sanos seleccionados, pesados y lavados con agua hervida tibia. Posteriormente, fueron picados junto con 4 gr. de ácido cítrico en 1 litro de agua. La cantidad total de fruta con la que se trabajó se obtuvo tras restar el peso del desperdicio con semillas del peso bruto inicial. Para realizar el licuado, se mezcló un litro de agua por cada kilogramo de fruta. Posteriormente, para refinar el producto y eliminar residuos se utilizó la máquina despulpadora. Adicionalmente se realizó un proceso de desinfección de la pulpa con $0,2 \mathrm{mg} \cdot \mathrm{L} \cdot{ }^{-1}$ de metabisulfito de sodio. Se dejó reposar durante 24 horas. Con ayuda del refractómetro se determinó el contenido de azúcar de cada variedad por tratamiento.

En la fermentación, se utilizaron envases de vidrio esterilizados con capacidad de un litro, donde se colocó un volumen de $600 \mathrm{ml}$. de pulpa a la que se adicionó 0.33 $\mathrm{ml}$ de levadura y $2,0 \mathrm{~g}$. de germen de trigo como fuente de nitrógeno y nutrientes para las levaduras. En el proceso de fermentación cada botella fue tapada con un corcho que posee un agujero en el medio por donde ingresó el equipo de venoclisis (trampa de aire), el cual permitió la eliminación de los gases. Las botellas fueron colocadas en un calentador a baño maría donde permanecieron a una temperatura de $21^{\circ} \mathrm{C} \pm 1^{\circ}$ durante 15 días. Posterior a ello, se filtró el contenido utilizando un lienzo y un embudo, de esta forma se eliminaron los sólidos presentes y parte de las levaduras muertas que se encontraban en el fondo.

Para desactivar a las levaduras vivas que pudiesen quedar y para desinfectar el vino se añadió $0,2 \mathrm{~g} \cdot \mathrm{L}^{-1}$ de metabisulfito. Como agente clarificante se aplicó 1 clara de huevo por cada 50 litros de vino, para ello se batió la clara con un poco de agua y 1 g. de sal. Se dosificó la mezcla a cada botella y dejó reposar hasta obtener un producto limpio y transparente. Al culminar el tiempo de clarificación se realizó un trasiego el que consistió en separar los sedimentos aún visibles que permanecen en la bebida como sólidos depositados al fondo del envase (Morris y Main, 2007).

Para subir el contenido de azúcar de los vinos a $12^{\circ}$ Brix, se añadió sirope que se obtiene de calentar una parte de agua por dos partes de azúcar (Larrea, 2015). Posteriormente se estabilizó el producto utilizando una solución de 0,48 g. de metabisulfito de potasio y 2,4 g. de ácido cítrico, la cual se distribuyó proporcionalmente a las botellas de vino. Finalmente, para mantener conservado el vino se procedió al embotellado. Las botellas de vino fueron sometidas al proceso de gasificación con hielo seco y otras al proceso térmico.

En coordinación con el presidente del GAD parroquial de Los Andes, Edgar Rodríguez, el 11 de diciembre de 2015 se realizó la difusión del proyecto de investigación con presencia de las autoridades y fruticultores de la mencionada parroquia (Figura 2). Allí se expusieron los conocimientos adquiridos, sobre el proceso de elaboración de bebidas de bajo grado alcohólico a base de durazno. Para que los catadores puedan reflejar sus juicios sobre un vino, compararlos y conservarlos, debe existir un procedimiento de expresión de resultados común para todos, para ello se utilizó la ficha de cata (Massini, 2007).

Posteriormente se realizó la evaluación sensorial de los vinos mediante el proceso de catación (Figura 3), en la cual los asistentes llenaron ficha de análisis sensorial recomendada por la Organización Internacional de la Viña y el Vino (OIV) y la Unión Internacional de Enólogos (UIOE), los vinos que tienen un puntaje entre 
100 - 88 puntos obtienen una calificación de Oro, entre 87 - 83 puntos una calificación de Plata y entre 82 - 76 puntos una calificación de Bronce, los que obtienen una calificación menor no son comerciales (Mijares \& Sáez, 2007).

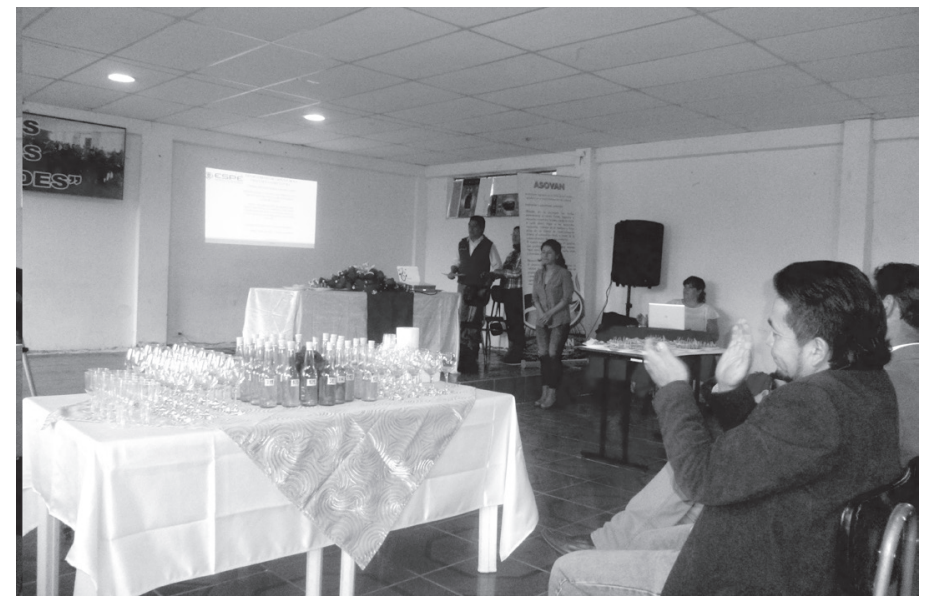

Figura 2. Capacitación sobre la elaboración del vino de durazno, por Viteri y Soria (2015)

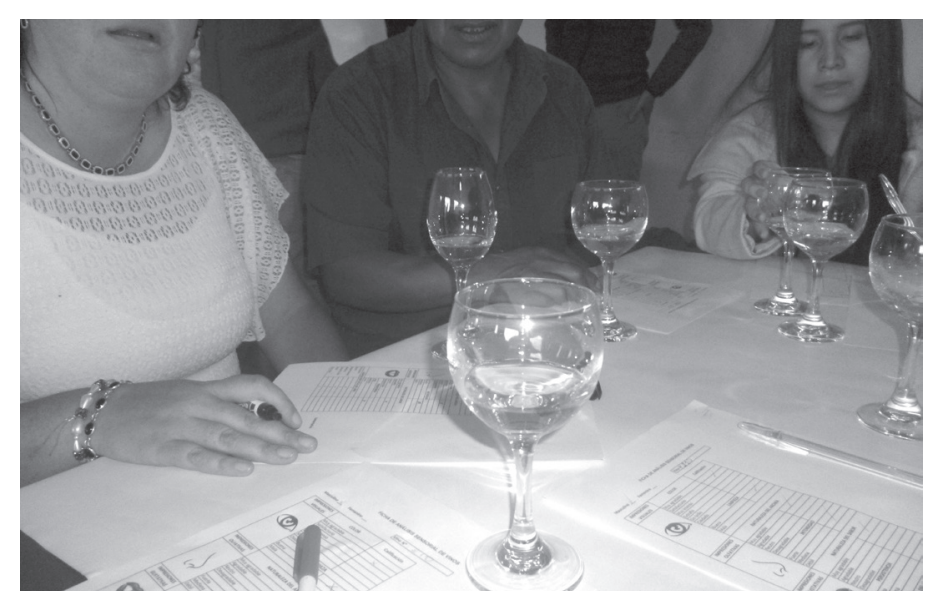

Figura 3. Capacitación del vino de durazno, por Viteri y Soria (2015)

\section{Resultados}

Se obtuvo un vino de mesa semiseco, con o sin gas, y libre de colorantes o sabores artificiales, que cumple con la normativa INEN N ${ }^{\circ} 371$ y 374, (INEN, 1987). El mayor contenido de azúcar se obtuvo con la variedad de durazno Abridor "Rosado de Sevilla" con un promedio de 13,53 ${ }^{\circ}$ Brix; y con la cepa de levadura Safale S-04 un promedio de $14,04^{\circ}$ Brix; el contenido promedio de azúcar en el día 133 fue de 5,33 por lo que se realizaron correcciones con sirope para igualar a $12^{\circ}$ Brix.

Se obtuvo un menor $\mathrm{pH}$ : con las variedades de durazno Abridor "Rosado de Sevilla" y Criollo con una media de 3,71 ; con el método de conservación térmica un promedio de 3,70; y los tratamientos que correspondían a la variedad Criollo con la cepa de levadura Safale S-04 alcanzaron un pH de 3,68.

El menor contenido de alcohol se consiguió con las variedades de durazno Abridor "Rosado de Sevilla" y Criollo, con un promedio de $8,06^{\circ}$ y $8,50{ }^{\circ} \mathrm{GL}$ respectivamente; la cepa de levadura Safale S-04 una media de $7,87^{\circ} \mathrm{GL}$; y con el método de conservación térmica un promedio de $8,35^{\circ} \mathrm{GL}$.

Se observó que existe una relación directa entre la limpieza del vino y los días transcurridos, es decir a mayor número de días los vinos adquirieron un color más traslúcido. El proceso de clarificación duró aproximadamente 3 meses.

Los resultados globales de la calificación cuantitativa obtenida de las encuestas realizadas a los tratamientos con la ficha de cata se observan en la Figura 4. Los tratamientos con mayor aceptabilidad en la evaluación sensorial fueron: para conservación carbonatada los que contenían la variedad de durazno Abridor "Rosado de Sevilla", mientras que en la conservación térmica los que correspondían a las variedades de durazno Abridor "Rosado de Sevilla" y Pepa de Oro. Los tratamientos que corresponden a la variedad de durazno Abridor "Rosado de Sevilla" obtuvieron una mejor aceptación en el proceso de catación, seguidos por la variedad Pepa de Oro, mientras que la variedad de durazno Criollo no fue evaluada puesto que no tuvo una clarificación final eficiente. Cuatro de los cinco tratamientos que obtuvieron una calificación de Oro fueron los que correspondían a la variedad de durazno Abridor "Rosado de Sevilla", y las cepas de levadura Safale S-04 y Cider Yeast; mientras que mayor puntaje obtuvieron los tratamientos con conservación carbonatada. 


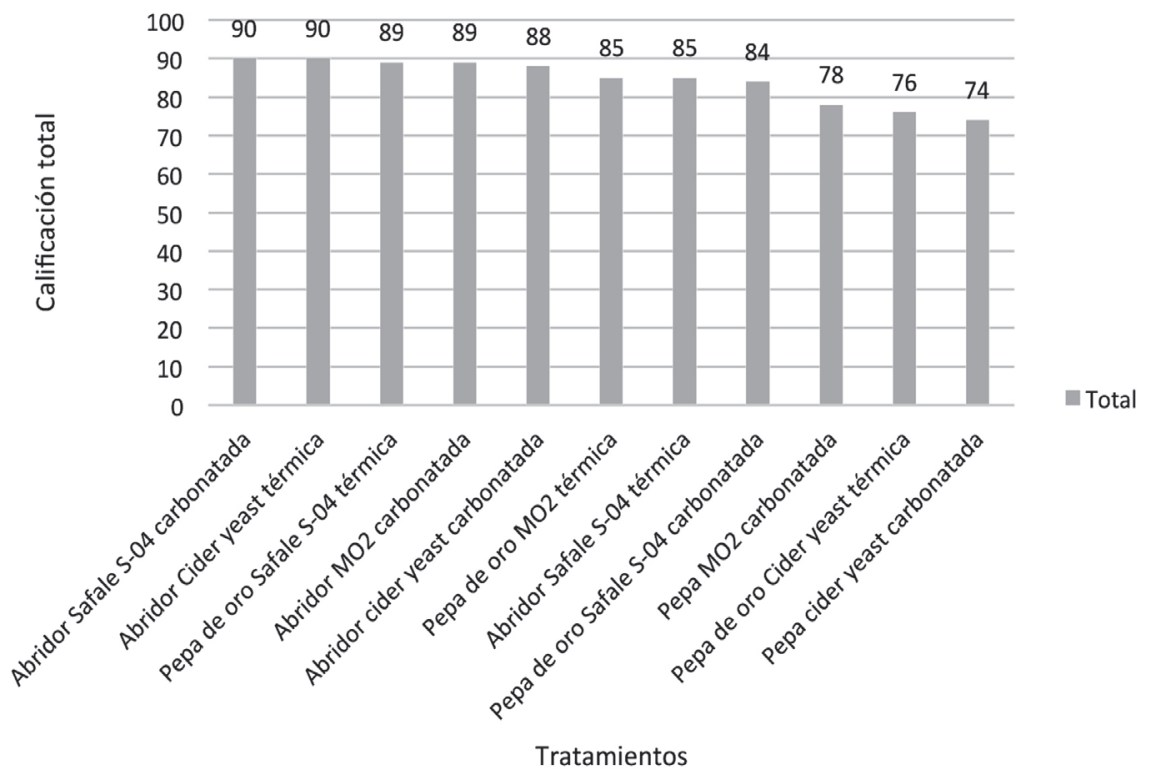

Figura 4. Calificación total cuantitativa de los tratamientos, por Viteri y Soria (2015)

\section{Conclusiones}

Se elaboró un producto con valor agregado, que a largo plazo mejorará la rentabilidad económica del fruto en la parroquia Los Andes- Patate, el durazno de la variedad Abridor "Rosado de Sevilla" fue el mejor representante en las pruebas de catación.

El mejor proceso fermentativo se obtuvo con la cepa de levadura Safale S-04, presentando un menor contenido de alcohol y $\mathrm{pH}$, y mayor cantidad de ${ }^{\circ}$ Brix. Además, en la catación de los vinos bajo este tratamiento la puntuación fue de Oro.

Los mayores puntajes en las pruebas de catación al final del tiempo necesario para su clarificación, los obtuvieron los tratamientos con conservación carbonatada.

\section{Referencias Bibliográficas}

Fermentis. (1 de Octubre de 2015). Fermentis Lesaffre for Beverages. Obtenido de Fermentis Web site: http://www.fermentis.com/wp-content uploads/2015/12/Safale-S-04-sp.pdf

Instituto Ecuatoriano de Normalización INEN. (3 de Agosto de 1987). INEN Servicio ecuatoriano de normalización. Obtenido de INEN Servicio ecuatoriano de normalización Sitio web: http://www.normalizacion.gob.ec/

Instituo Ecuatoriano de Normalización INEN. (1 de Julio de 1987). INEN Servicio ecuatoriano de normalización. Obtenido de INEN Servicio ecuatoriano de normalización Sitio web: http://www.normalizacion.gob.ec/

Larrea, G. (3 de Noviembre de 2015). Fórmula para corregir el contenido de azúcar en los vinos. (M. Viteri, Entrevistador)

Linde. (2005). The Linde group. Recuperado el 7 de Noviembre de 2015, de Linde sitio web: ttp://www.linde-gas.com.ar/international/web/lg/ar/likelgar.nsf/ docbyalias/nav app hielo seco

Mad Millie. (2011). Mad millie: as fresh as can be. Obtenido de Mad millie Web site: http://www.madmillie.com/shop/Cider/Consumables/Cider+Yeast.html

Mangrove Jack`s. (2015). Mangrove Jack `s. Obtenido de http://mangrovejacks.com/ Massini, G. (2007). El arte de la cata. Barcelona: Robinbook. Recuperado el 1 de Diciembre de 2015, de https://books.google.com.ec/ books? id $=$ CG5YBsYFdGgC\&printsec $=$ frontcover\&hl=es\&source $=\mathrm{gbs}$ ge_summary_r\&cad $=0 \# \mathrm{v}=$ onepage $\& \mathrm{q} \& \mathrm{f}=$ false

Mijares, M. I., \& Sáez, J. A. (2007). El vino de la cepa a la copa (Cuarta ed.). Madrid: Ediciones Mundi-Prensa. Obtenido de https://books.google.com. ec

Morris, J.,\& Main, G. (2007). Clarificantes para vino. Obtenido deAlimentaria Online: http://www.alimentariaonline.com/media/MA018_CLARIVINO_F.pdf

Plan de Ordenamiento Territorial Patate PDOT - PATATE. (2011). Principales cultivos. 3-10. Patate - Ecuador.

Pérez, C., \& Gervás, J. (2010). Elaboración artesanal del vino: manual práctico de la vida autosuficiente (Séptima ed.). BLUME. 